\title{
Impact of Molecular Subtypes in Muscle-invasive Bladder Cancer on Predicting Response and Survival after Neoadjuvant Chemotherapy
}

\begin{abstract}
Roland Seiler $^{a, b}$, Hussam Al Deen Ashab ${ }^{c}$, Nicholas Erho $^{c}$, Bas W.G. van Rhijn ${ }^{d}$, Brian Winters ${ }^{e}$, James Douglas $^{f}$, Kim E. Van Kessel ${ }^{g}$, Elisabeth E. Fransen van de Putte ${ }^{d}$, Matthew Sommerlad ${ }^{f}$, Natalie Q. Wang ${ }^{c}$, Voleak Choeurng ${ }^{c}$, Ewan A. Gibb ${ }^{c}$, Beatrix Palmer-Aronsten ${ }^{c}$, Lucia L. Lam ${ }^{c}$, Christine Buerki $^{c}$, Elai Davicioni ${ }^{c}$, Gottfrid Sjödahl $^{h}$, Jordan Kardos $^{i}$, Katherine A. Hoadley ${ }^{i}$, Seth P. Lerner ${ }^{j}$, David J. McConkey ${ }^{k}$, Woonyoung Choi ${ }^{k}$, William Y. Kim ${ }^{i}$, Bernhard Kiss ${ }^{b}$, George N. Thalmann ${ }^{b}$, Tilman Todenhöfer ${ }^{a}$, Simon J. Crabb ${ }^{l}$, Scott North ${ }^{m}$, Ellen C. Zwarthoff ${ }^{g}$, Joost L. Boormans ${ }^{n}$, Jonathan Wright ${ }^{e}$, Marc Dall'Era $^{\circ}$, Michiel S. van der Heijden $^{d}$, Peter C. Black ${ }^{a, *}$

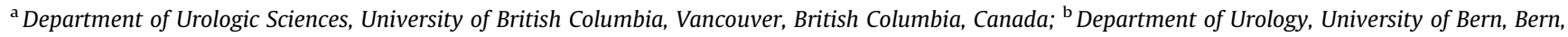
Switzerland; ' GenomeDx Biosciences, Inc., Vancouver, British Columbia, Canada; ${ }^{\mathrm{d}}$ Department of Surgical Oncology, Division of Urology, Netherlands Cancer Institute-Antoni van Leeuwenhoek Hospital, Amsterdam, The Netherlands; ${ }^{\mathrm{e}}$ Department of Urology, University of Washington School of Medicine, Seattle, Washington, USA; ${ }^{\mathrm{f}}$ Department of Urology, University Hospital of Southampton, Hampshire, UK; ${ }^{\mathrm{g}}$ Department of Pathology, Erasmus MC, University Medical Center Rotterdam, Rotterdam, The Netherlands; ${ }^{\mathrm{h}}$ Division of Urological Research, Department of Translational Medicine, Lund University, Malmö, Sweden; ${ }^{\mathrm{i}}$ Lineberger Comprehensive Cancer Center, University of North Carolina at Chapel Hill, Chapel Hill, North Carolina, USA; ${ }^{\mathrm{j}}$ Scott Department of Urologic Oncology, Baylor College of Medicine, Houston, Texas, USA; ${ }^{\mathrm{k}}$ Department of Urology, University of Texas MD Anderson Cancer Center, Houston, Texas, USA; ${ }^{1}$ Department of Medical Oncology, University Hospital of Southampton, Hampshire, UK; ${ }^{\mathrm{m}}$ Cross Cancer Institute, Department of Oncology, University of Alberta Edmonton, Alberta, Canada; ${ }^{\mathrm{n}}$ Department of Urology, Erasmus MC, University Medical Center Rotterdam, Rotterdam, The Netherlands; ${ }^{\circ}$ UC Davis Comprehensive Cancer Center, Sacramento, California, USA
\end{abstract}

\section{Article info}

Article history:

Accepted March 21, 2017

Associate Editor:

James Catto

Keywords:

Bladder cancer

Neoadjuvant chemotherapy

Molecular subtypes

Response prediction

\begin{abstract}
Background: An early report on the molecular subtyping of muscle-invasive bladder cancer (MIBC) by gene expression suggested that response to neoadjuvant chemotherapy (NAC) varies by subtype.

Objective: To investigate the ability of molecular subtypes to predict pathological downstaging and survival after NAC.

Design, setting, and participants: Whole transcriptome profiling was performed on preNAC transurethral resection specimens from 343 patients with MIBC. Samples were classified according to four published molecular subtyping methods. We developed a single-sample genomic subtyping classifier (GSC) to predict consensus subtypes (claudin-low, basal, luminal-infiltrated and luminal) with highest clinical impact in the context of NAC. Overall survival (OS) according to subtype was analyzed and compared with OS in 476 non-NAC cases (published datasets).

Intervention: Gene expression analysis was used to assign subtypes.
\end{abstract}

\section{EU *ACME}

www.eu-acme.org/ europeanurology

\footnotetext{
* Corresponding author. 2775 Laurel St., Vancouver, British Columbia V5Z 1M9, Canada. Tel. +1 604875 4301; Fax: +1 6048755604. E-mail address: pblack@mail.ubc.ca (P.C. Black).
} 
Please visit www.eu-acme.org/ europeanurology to read and answer questions on-line. The EU-ACME credits will then be attributed automatically.
Outcome measurements and statistical analysis: Receiver-operating characteristics were used to determine the accuracy of GSC. The effect of GSC on survival was estimated by Cox proportional hazard regression models.

Results and limitations: The models generated subtype calls in expected ratios with high concordance across subtyping methods. GSC was able to predict four consensus molecular subtypes with high accuracy (73\%), and clinical significance of the predicted consensus subtypes could be validated in independent NAC and non-NAC datasets. Luminal tumors had the best OS with and without NAC. Claudin-low tumors were associated with poor OS irrespective of treatment regimen. Basal tumors showed the most improvement in OS with NAC compared with surgery alone. The main limitations of our study are its retrospective design and comparison across datasets.

Conclusions: Molecular subtyping may have an impact on patient benefit to NAC. If validated in additional studies, our results suggest that patients with basal tumors should be prioritized for NAC. We discovered the first single-sample classifier to subtype MIBC, which may be suitable for integration into routine clinical practice.

Patient summary: Different molecular subtypes can be identified in muscle-invasive bladder cancer. Although cisplatin-based neoadjuvant chemotherapy improves patient outcomes, we identified that the benefit is highest in patients with basal tumors. Our newly discovered classifier can identify these molecular subtypes in a single patient and could be integrated into routine clinical practice after further validation.

(C) 2017 European Association of Urology. Published by Elsevier B.V. All rights reserved.

\section{Introduction}

Neoadjuvant cisplatin-based chemotherapy (NAC) is the standard treatment in muscle-invasive bladder cancer (MIBC) prior to radical cystectomy [1-3]. Although NAC improves pathological downstaging and patient survival, only approximately $40 \%$ of patients experience a major response, defined as absence of muscle-invasive disease and lymph node metastasis ( $<$ pT2 and pN0) [4]. Nonresponding patients are unlikely to derive clinical benefit, are exposed to substantial toxicity, and experience a delay in definitive local therapy [2,3]. Identification of molecular markers of nonresponsiveness is essential for more precise delivery of care. Recent analyses suggest that specific mutations, especially in ERCC2, ERBB2, and DNA repair genes, may predict response to NAC [5-8]. Here we aimed to use RNA expression analysis for the development of predictive biomarkers.

Recent identification of molecular gene expression subtypes [9-13] and prior work highlighting the clinical impact of basal MIBC [14] have advanced our understanding of the biology of bladder cancer. Molecular classification provides a framework for further study and has potential implications for the clinical management of MIBC. Four different molecular subtyping schemes have been described [10-13]. Each was developed in different patient populations using unique genomic platforms, and only one was based on integrative multiplatform genomic analysis [10]. Despite these differences, each identifies molecular phenotypes that share many similarities. They represent a division into basal and luminal tumors at a higher level, with different subclassifications that are specific to each system.

Choi et al [11] first introduced the concept that molecular subtypes may predict response to NAC. In three cohorts with a total of 100 patients, a subset classified as having "p53-like" tumors demonstrated a lower response rate to cisplatin-based combination chemotherapy. This finding has not been validated in additional larger patient cohorts and has not been investigated with the other subtyping methods. Furthermore, none of the four subtyping models is suitable for clinical implementation because each requires classification of an entire patient cohort in order to assign an individual patient sample to a subtype.

In this study, we aimed to correlate large multiinstitutional patient cohort outcomes after NAC with molecular subtyping of pre-NAC specimens according to four published classification methods: University of North Carolina (UNC), MD Anderson Cancer Center (MDA), The Cancer Genome Atlas (TCGA), and Lund University [1013,15]. Moreover, we aimed to develop a single-patient assay based on transcriptomic analysis of transurethral resection (TUR) specimens that would be suitable for use in a clinical laboratory setting.

\section{Patients and methods}

\subsection{Patient populations}

For the discovery NAC cohort, 250 consecutive patients from five institutions were compiled. MIBC (cT2-4aN0-3M0) was diagnosed by TUR prior to receiving at least three cycles of NAC. For the validation NAC cohort, 93 consecutive patients with MIBC from two institutions were selected, whose characteristics were similar to those of the discovery set.

\subsection{Tissue sampling and gene expression profiling}

Whole transcriptome analysis was performed on formalin-fixed, paraffin-embedded tumor tissue with GeneChip ${ }^{\circledR}$ Human Exon 1.0 ST Array (Affymetrix) in a Clinical Laboratory Improvement Amendments (CLIA)-certified laboratory [16]. In total, 223/250 (89\%) and 82/93 (88\%) of the discovery and validation NAC cohorts, respectively, passed quality control (Supplementary Table 1). Microarray data were normalized and genes summarized using single-channel array normalization [17].

\subsection{Datasets from the public domain}

For investigation of the prognostic impact of the published methods for molecular subtyping, the 397 patients without chemotherapy prior to sample collection from the TCGA bladder urothelial carcinoma were 
Table 1 - Patient characteristic table of the cisplatin-based NAC set $(n=269)$

\begin{tabular}{|c|c|c|c|c|c|c|c|c|c|c|}
\hline Variable & & Bern & NKI & UHS & UW & VGH & $\mathrm{UCD}^{\mathrm{a}}$ & $\mathrm{EMC}^{\mathrm{a}}$ & Total & $p$ value \\
\hline$n$ & & 40 & 49 & 22 & 41 & 48 & 26 & 43 & 269 & \\
\hline $\begin{array}{l}\text { Overall survival time } \\
\text { (censored patients) }\end{array}$ & Median (IQR) & $48(26-73)$ & $46(31-87)$ & $49(40-59)$ & $48(26-61)$ & $31(13-41)$ & $9(4-23)$ & $24(13-36)$ & $35(16-54)$ & - \\
\hline Age & Mean (SD) & $64(10)$ & $57(11)$ & $67(12)$ & $62(11)$ & $61(9)$ & $65(8)$ & $59(13)$ & $61(11)$ & - \\
\hline \multirow[t]{2}{*}{ Gender (\%) } & Female & $12(30.0)$ & $17(34.7)$ & $7(31.8)$ & $10(24.4)$ & $14(29.2)$ & $8(30.8)$ & $12(27.9)$ & $80(29.7)$ & 0.97 \\
\hline & Male & $28(70.0)$ & $32(65.3)$ & $15(68.2)$ & $31(75.6)$ & $34(70.8)$ & $18(69.2)$ & $31(72.1)$ & $189(70.3)$ & \\
\hline \multirow{5}{*}{$\begin{array}{l}\text { Clinical tumor } \\
\text { stage (\%) }\end{array}$} & $\mathrm{T} 1$ & $1(2.5)$ & $0(0.0)$ & $0(0.0)$ & $0(0.0)$ & $0(0.0)$ & $0(0.0)$ & $0(0.0)$ & $1(0.4)$ & $<0.001$ \\
\hline & T2 & $4(10.0)$ & $10(20.4)$ & $18(81.8)$ & $20(48.8)$ & $26(54.2)$ & $25(96.2)$ & $17(39.5)$ & $120(44.6)$ & \\
\hline & T3 & $21(52.5)$ & $26(53.1)$ & $4(18.2)$ & 13 (31.7) & 19 (39.6) & $1(3.8)$ & 18 (41.9) & $102(37.9)$ & \\
\hline & $\mathrm{T} 4$ & $14(35.0)$ & $13(26.5)$ & $0(0.0)$ & $8(19.5)$ & $3(6.2)$ & $0(0.0)$ & $4(9.3)$ & $42(15.6)$ & \\
\hline & NA & $0(0.0)$ & $0(0.0)$ & $0(0.0)$ & $0(0.0)$ & $0(0.0)$ & $0(0.0)$ & $4(9.3)$ & $4(1.5)$ & \\
\hline \multirow{3}{*}{$\begin{array}{l}\text { Clinical lymph node } \\
\text { stage (\%) }\end{array}$} & No & $14(35.0)$ & $21(42.9)$ & $20(90.9)$ & $33(80.5)$ & $38(79.2)$ & $0(0.0)$ & $32(74.4)$ & $158(58.7)$ & $<0.001^{\mathrm{b}}$ \\
\hline & N1-3 & $26(65.0)$ & $28(57.1)$ & $2(9.1)$ & $8(19.5)$ & $10(20.8)$ & $0(0.0)$ & $7(16.3)$ & $81(30.1)$ & \\
\hline & NA & $0(0.0)$ & $0(0.0)$ & $0(0.0)$ & $0(0.0)$ & $0(0.0)$ & $26(100.0)$ & $4(9.3)$ & $30(11.2)$ & \\
\hline \multirow{5}{*}{$\begin{array}{l}\text { Pathological tumor } \\
\text { stage (\%) }\end{array}$} & pT0/is/a & $13(32.5)$ & $24(49.0)$ & $9(40.9)$ & $18(43.9)$ & 19 (39.6) & $9(34.6)$ & $13(30.2)$ & 105 (39.0) & 0.618 \\
\hline & pT1 & $3(7.5)$ & $2(4.1)$ & $0(0.0)$ & $5(12.2)$ & $2(4.2)$ & $2(7.7)$ & $6(14.0)$ & $20(7.4)$ & \\
\hline & pT2 & $8(20.0)$ & $7(14.3)$ & $5(22.7)$ & $6(14.6)$ & $13(27.1)$ & $3(11.5)$ & $5(11.6)$ & $47(17.5)$ & \\
\hline & рT3/4 & $16(40.0)$ & $15(30.6)$ & $8(36.4)$ & $12(29.3)$ & $14(29.2)$ & $11(42.3)$ & $18(41.9)$ & $94(34.9)$ & \\
\hline & NA & $0(0.0)$ & $1(2.0)$ & $0(0.0)$ & $0(0.0)$ & $0(0.0)$ & $1(3.8)$ & $1(2.3)$ & $3(1.1)$ & \\
\hline \multirow{3}{*}{$\begin{array}{l}\text { Pathological lymph } \\
\text { node stage (\%) }\end{array}$} & pNO & $24(60.0)$ & $28(57.1)$ & $18(81.8)$ & $33(80.5)$ & $41(85.4)$ & $16(61.5)$ & $35(81.4)$ & $195(72.5)$ & 0.04 \\
\hline & pN1-3 & $16(40.0)$ & $12(24.5)$ & $4(18.2)$ & $8(19.5)$ & $6(12.5)$ & $8(30.8)$ & $7(16.3)$ & $61(22.7)$ & \\
\hline & NA & $0(0.0)$ & $9(18.4)$ & $0(0.0)$ & $0(0.0)$ & $1(2.1)$ & $2(7.7)$ & $1(2.3)$ & $13(4.8)$ & \\
\hline \multirow[t]{3}{*}{ NAC type (\%) } & GemCis & $40(100.0)$ & $16(32.7)$ & $22(100.0)$ & $22(53.7)$ & $48(100.0)$ & $19(73.1)$ & $33(76.7)$ & $200(74.3)$ & $<0.001$ \\
\hline & MVAC & $0(0.0)$ & $33(67.3)$ & $0(0.0)$ & $19(46.3)$ & $0(0.0)$ & $6(23.1)$ & $5(11.6)$ & $63(23.4)$ & \\
\hline & Other & $0(0.0)$ & $0(0.0)$ & $0(0.0)$ & $0(0.0)$ & $0(0.0)$ & $1(3.8)$ & $5(11.6)$ & $6(2.2)$ & \\
\hline $\begin{array}{l}\text { GSC = genomic subty } \\
\text { NAC = neoadjuvant ch } \\
\text { a } U C D \text { and EMC sets } r \\
\text { b Excluding UCD due }\end{array}$ & $\begin{array}{l}\text { sent blinde } \\
\text { ck of clinica }\end{array}$ & mph no & $\begin{array}{l}\text { alidation } \\
\text { atus. }\end{array}$ & not use & 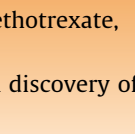 & e GSC. & & ind cisp & ; $\quad \mathrm{NA}=$ not & pplicable; \\
\hline
\end{tabular}

used. As an unknown proportion may have subsequently received NAC, validation of the discovered single-sample classifier was performed only in those 179 without chemotherapy prior to or after sample collection. For validation of the discovered classifier, two datasets containing 107 (MDA, GSE48075) and 190 (Lund University, GSE32894) patients with MIBC who did not receive NAC were downloaded from Gene Expression Omnibus database.

\subsection{Assignment to subtypes}

The group from UNC provided the classifiers to assign the samples to claudin-low, basal, and luminal subtypes $[12,15]$. The one nearestneighbor prediction model from the MDA group assigned the tumors to basal, p53-like, and luminal subtypes [11]. The model based on classification to nearest centroids was used to assign the tumors to the TCGA clusters [10]. The group from Lund provided the centroid values for each subtype of their original model [13].

\subsection{Discovery and validation of the single-sample classifier}

A detailed description of the model development, model object, and score generation code are provided in the supplementary material. In brief, we developed a single-sample model to predict four subtypes (claudin-low, basal, luminal-infiltrated, and luminal). A generalized linear model with elastic net regularization (GLMNET) was trained.

\subsection{Statistical analyses}

Statistical analyses are described in the supplementary information.

\section{Results}

Clinicopathological details of the discovery and validation NAC metadatasets are provided in Table 1 and Supplementary Table 1.

\subsection{Subtyping calls of the published models in the discovery NAC dataset}

The frequency of each subtype in all pre-NAC samples $(n=223)$ was in expected ratios $[10,15]$ (Fig. 1A). Gene expression of given biological functions confirmed the concordance between the different subtyping methods (Fig. 1B, Supplementary Fig. 1, and Supplementary Table 2). For example, all luminal subtypes showed higher expression of genes for urothelial differentiation (Fig. 1B, Supplementary Fig. 2 and Supplementary Fig. 3) compared with all basal subtypes. T-cell and myeloid cell signatures were highest in the expected subtypes (claudin-low [UNC] and cluster IV [TCGA]). Genes from the extracellular matrix were highly expressed in p53like (MDA; Supplementary Fig. 3E) and infiltrated subtypes (Lund; Fig. 1B). Taken together, these results demonstrate high concordance between the subtyping methods, representing the biological spectrum of MIBC as reported previously. 
A

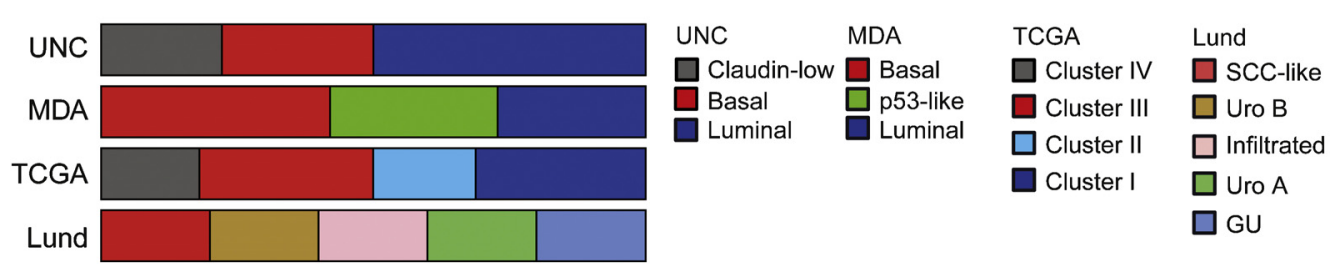

B

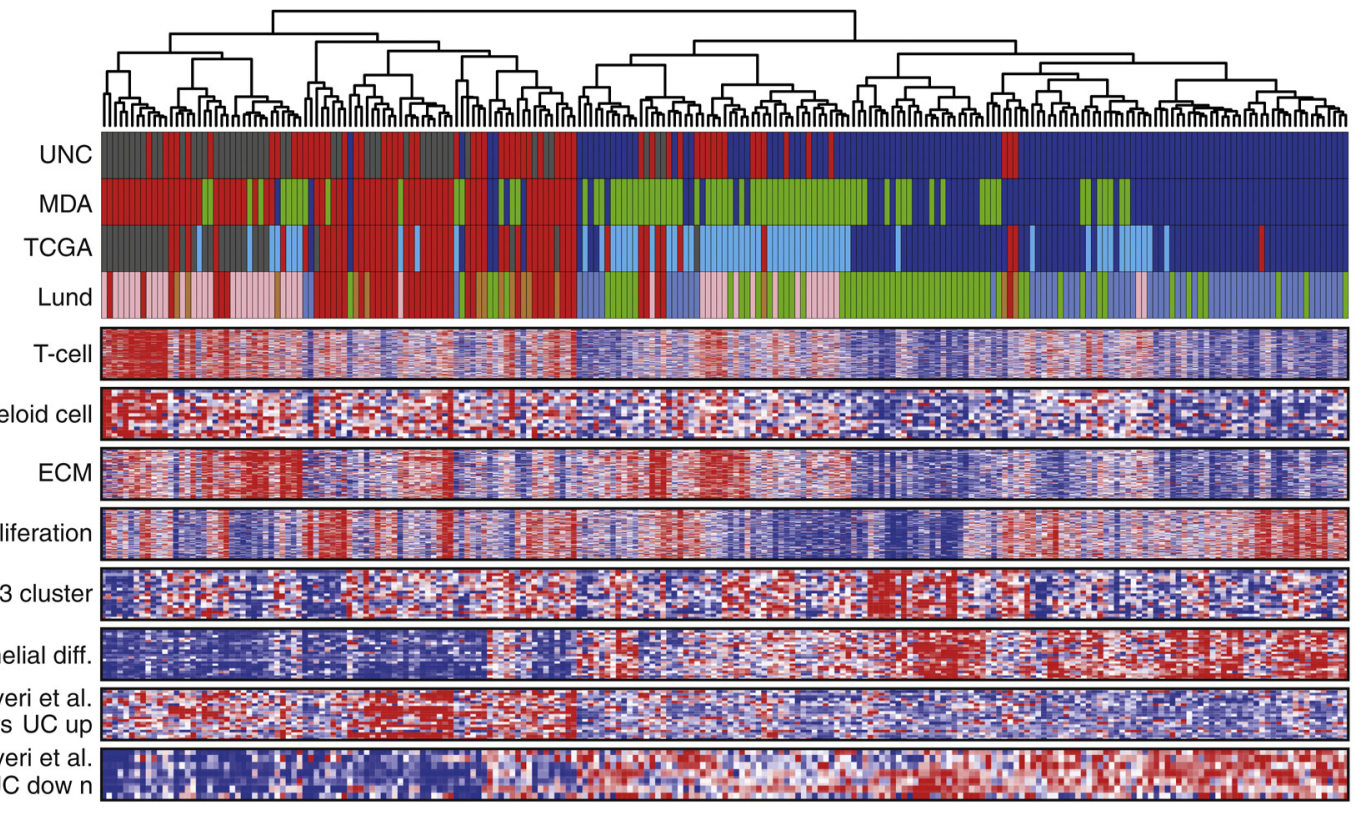

C

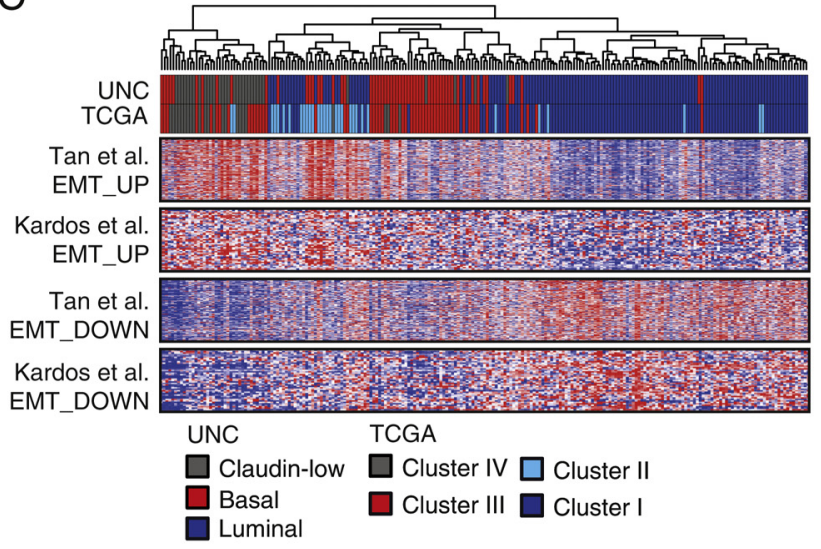

E

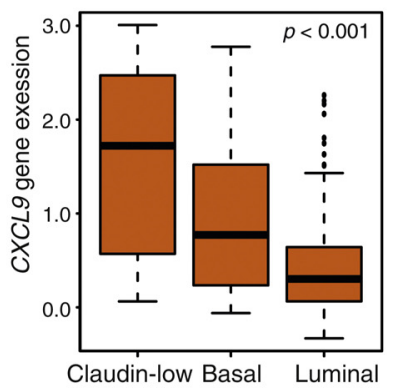

D

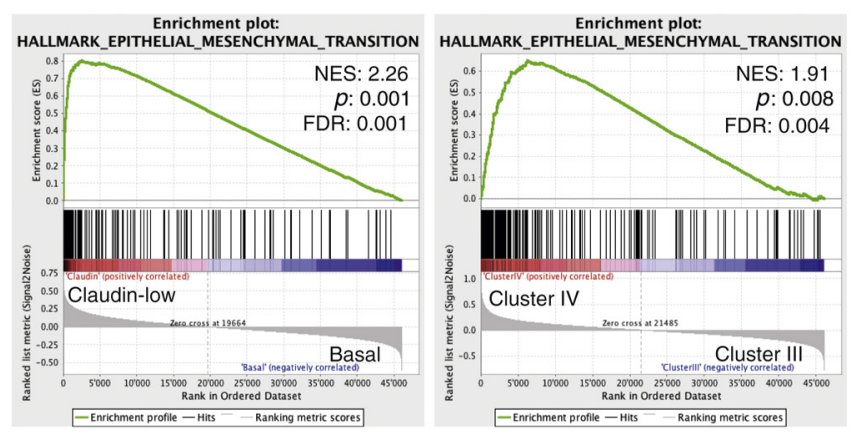

Fig. 1 - Molecular subtypes in pre-NAC TURBT samples in discovery NAC cohort. (A) Overview of four previously described bladder cancer subtyping methods. UNC divides the cancers in two classes, luminal and basal. Claudin-low is a subtype within basal that has lost epithelial differentiation and has a high level of immune infiltration. The MDA subtyping method also divides into basal and luminal tumors, in addition to a third subtype with an 


\subsection{Similarities between subtypes across different methods}

As recently reported, claudin-low tumors have basal characteristics with high expression levels of genes indicative of epithelial-to-mesenchymal transition (EMT) and immune infiltration (Fig. 1C-E, and Supplementary Fig. 4A and 4C). Tumors in TCGA cluster IV identify a similar subset of basal tumors: $21 / 28$ cluster IV tumors were labeled claudin-low. Cluster IV showed enrichment for EMT, chemokine signaling, and immune infiltration compared with cluster III tumors (Fig. 1C, 1D, 1F, and Supplementary Fig. 4B and 4D). Cluster II represents luminal tumors that are immune infiltrated [18]. Compared with cluster I tumors, cluster II tumors were EMT-signature positive and enriched for inflammatory response and chemokine signaling (Fig. 1F, and Supplementary Fig. 4B and 4E).

\subsection{Patient benefit from NAC differs between subtypes}

Extravesical extension of residual primary tumor (pT3/4) after NAC was observed more frequently in claudin-low (49\%), p53-like (38\%), and cluster II (40\%) subtypes. None of the subtypes was associated with a major response to NAC (ypT $<2 \mathrm{NO}$ ) on final pathology (Supplementary Table 3).

Overall survival (OS) varied by molecular subtype and between non-NAC and NAC patients (Fig. 2). By all subtyping methods, non-NAC-treated patients (TCGA cohort) with basal tumors had worse outcomes compared with those luminal tumors (Fig. 2). Of both luminal TCGA clusters, patients with cluster II tumors experienced worse OS than those with cluster I tumors. Poor OS was also observed in patients with claudin-low tumors.

The association between subtype and prognosis differed in our NAC cohort. The most pronounced difference was in patients with basal or equivalent subtypes (cluster III, urobasal [Uro] B, and squamous cell carcinoma [SCC]-like), who experienced a dramatic improvement in OS after NAC compared with the TCGA patients who received no NAC (Fig. 2). However, there was no such shift in survival in patients with claudin-low and cluster IV tumors, indicating that these patients fared poorly regardless of NAC even though they are subsets of the basal subtype. Across the different subtyping methods, patients with luminal or equivalent tumors had the best OS with or without NAC, with the exception of cluster II patients, who fared poorly in both settings, as did patients with p53-like tumors. There was no noteworthy difference in these trends when the patients were analyzed according to NAC regimen (gentacibine vs methotrexate, vinblastine, adriamycin, and cisplatin; Supplementary Fig. 5).

In summary, the differences in OS by subtype and the apparent impact of NAC suggest that a classification into four subtypes would have the greatest clinical relevance. Basal tumors warrant subclassification into tumors without and with EMT and immune infiltration (ie, basal and claudin-low, respectively), since NAC appeared to have the greatest impact on noninfiltrated basal tumors. Luminal tumors similarly warrant subclassification into tumors without and with EMT and immune infiltration (ie, luminal and luminal-infiltrated, respectively), since OS differed between the two groups.

\subsection{Single-sample classifier to predict bladder cancer subtypes}

Based on the biological characteristics and different impacts on clinical outcome, we trained a single-sample genomic subtyping classifier (GSC) to predict four classes based on the consensus of the different classification schemes: claudinlow, basal, luminal-infiltrated, and luminal (Fig. 3A and Supplementary Table 4). Compared with previously published methods, the single-sample GSC was more discriminate in assigning individual patients to a definitive subtype, as seen by the number of patients who have a dominant subtype score (Fig. 3B and Supplementary Fig. 6). Under 10fold cross validation, the overall accuracy of GSC assignment to the four classes in the discovery cohort was 76\% $(n=223)$. In the validation cohort $(n=82)$, the accuracy was $73 \%$ and significantly higher $(p<0.001)$ compared with the noinformation rate of $39 \%$ (defined by assignment of the subtype based on majority class). A multinomial goodnessof-fit test comparing the predicted probabilities from the GSC with the consensus subtype classes using the validation cohort found that the independent set was well predicted by the model and not significantly different compared with the discovery cohort $(p=0.47)$. Furthermore, in both the NAC and the non-NAC cohort $(n=476)$, consensus subtype classes (ie, those obtained using previously published clustering-based approaches) were all predicted with areas under the curve $>0.85$ (Fig. 3C).

\subsection{Prediction of clinical endpoints using GSC}

In both NAC datasets, we used only cases that were treated with cisplatin-based NAC for analysis of outcomes. The clinical significance of the predicted classes in the NAC datasets was compared with an independent non-NAC

\footnotetext{
active p53 signature (p53-like). The TCGA subtyping defines four clusters that are also basal (clusters III and IV) and luminal (clusters I and II). The Lund group discovered five subtypes that can be considered basal (Uro B and SCC-like), luminal (Uro A and genetically unstable), and infiltrated. (B) Heatmap of biologically relevant gene signatures (rows) in pre-NAC TUR samples from the discovery cohort. The column annotation across the top provides the subtype calls from each classification system. Claudin-low and cluster IV tumors showed the highest expression of the T-cell and myeloid cell signatures. Genes expressed in the ECM are expressed in the p53-like and infiltrated subtypes. Proliferation markers were highly expressed in genomically unstable tumors, while the Uro A subtype expressed an FGFR3 signature. Luminal tumors across subtyping methods express urothelial differentiation genes more highly. (C) Heatmap of two bidirectional EMT signatures (Tan et al and Kardos et al [15]). Claudin-low and cluster IV tumors both were EMT-signature positive, and cluster II tumors showed more EMT than cluster I tumors. (D) Enrichment plots of the hallmark EMT signature in claudin-low versus basal tumors (left) and cluster IV versus III (right). Both subgroups showed significant enrichment of EMT markers. Differential expression of immune markers (E) CXCL9 and (F) CD8A in the UNC subtypes (left) and the TCGA clusters (right). The claudin-low and cluster IV tumors showed the highest expression of immune markers. Reference levels: claudin-low, cluster IV. Diff. = differentiation; ECM = extracellular matrix; EMT = epithelial-to-mesenchymal transition; $G U$ = genomically unstable; MDA = MD Anderson Cancer Center; NAC = neoadjuvant chemotherapy; $S C C=$ squamous cell carcinoma; TCGA = The Cancer Genome Atlas; TUR = transurethral resection; TURBT = transurethral resection of bladder tumor; UNC = University of North Carolina; Uro = urobasal.
} 
A

Non-NAC TCGA dataset $(n=397)$

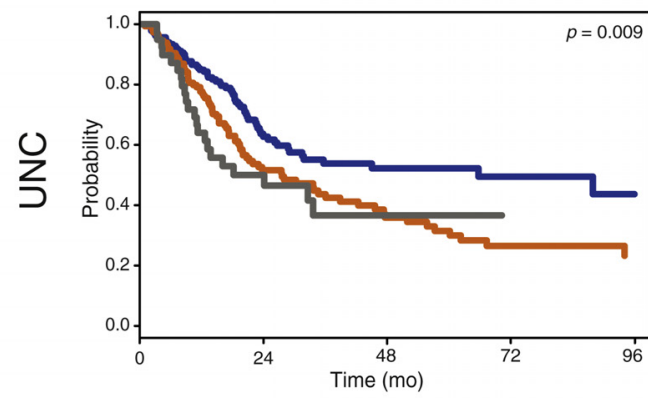

Claudin $\square 41$

Basal $\square 158$

Luminal $\square 195$

B

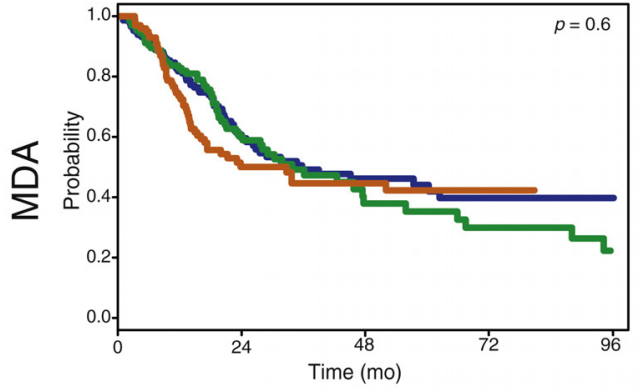

Basal $\square 106$

p53-like $\square 136$

Luminal $\square 152$

C
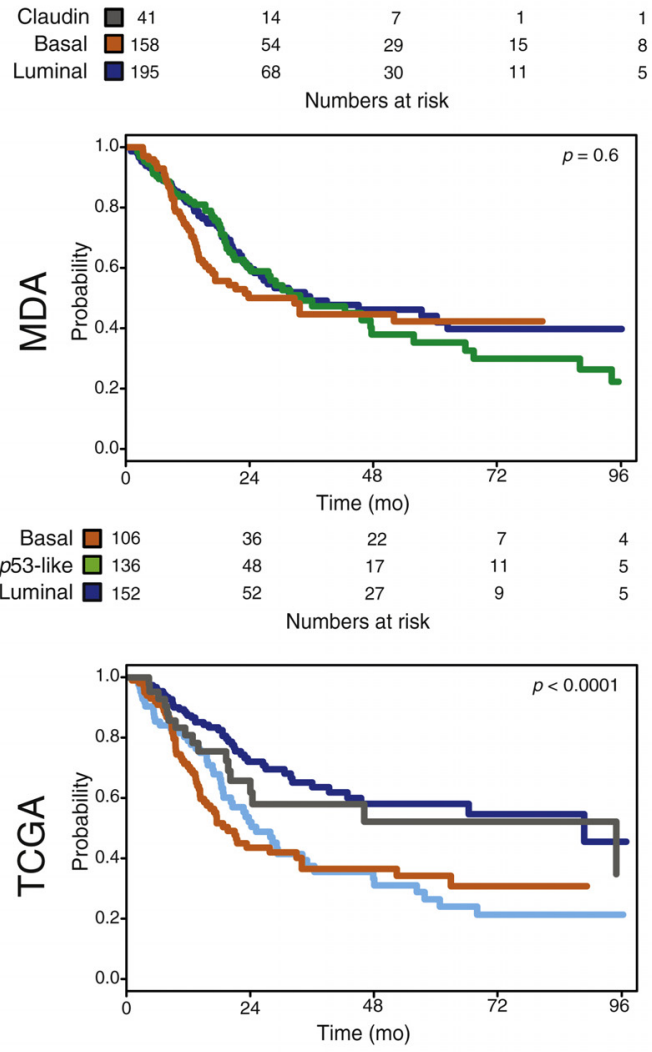

$\begin{array}{ll}1 & \square 41 \\ 8 & \square 52 \\ 5 & \square 107\end{array}$

Discovery NAC dataset $(n=200)$
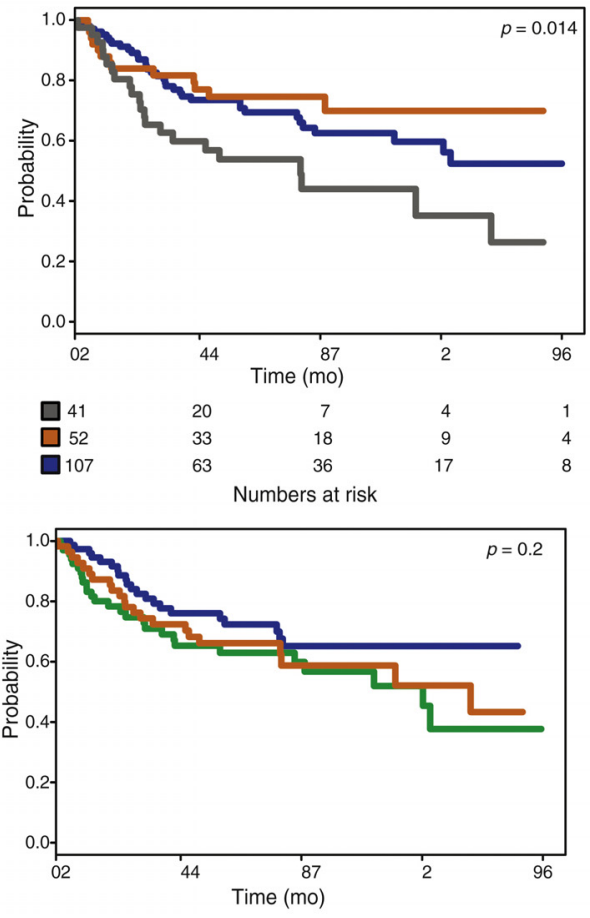

\section{$\square 1$}

$\square 52$

$\square 107$
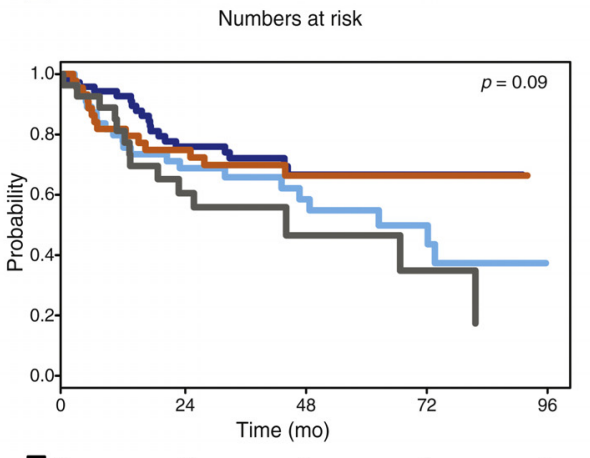

Cluster III $\square 103$

Cluster II $\square 86$

Cluster I 161

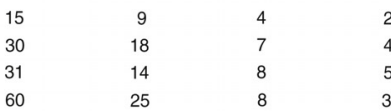

Numbers at risk

D
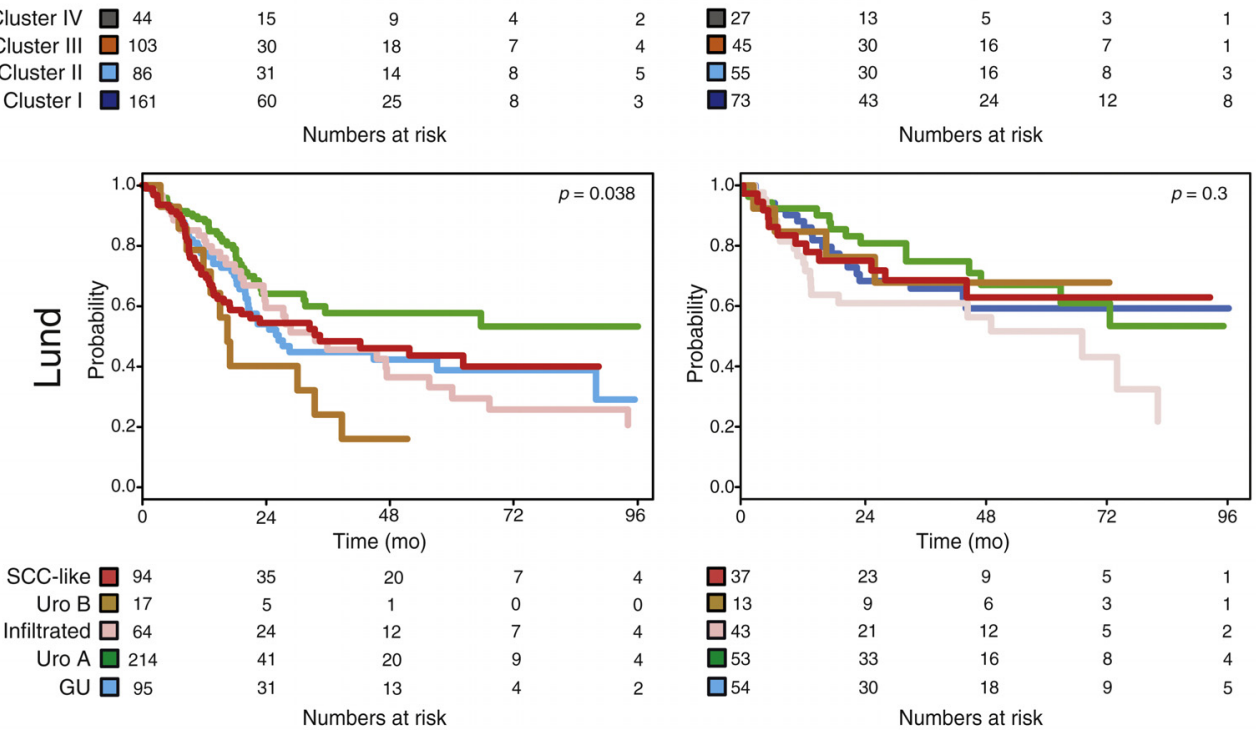

Fig. 2 - Kaplan-Meier estimates of OS according to molecular subtype in the non-NAC (left) and NAC (right) discovery datasets. (A) OS stratified according to the UNC subtypes. In the non-NAC setting (TCGA, left), patients with claudin-low and basal tumors had worse OS compared to patients with luminal tumors. The prognostic significance of each subtype changed in the context of NAC (right). While patients with claudin-low tumors still 
A

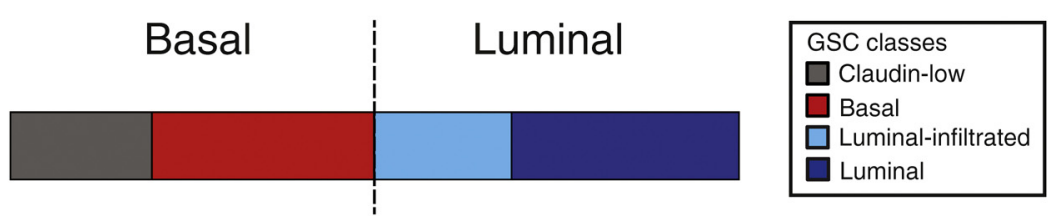

B

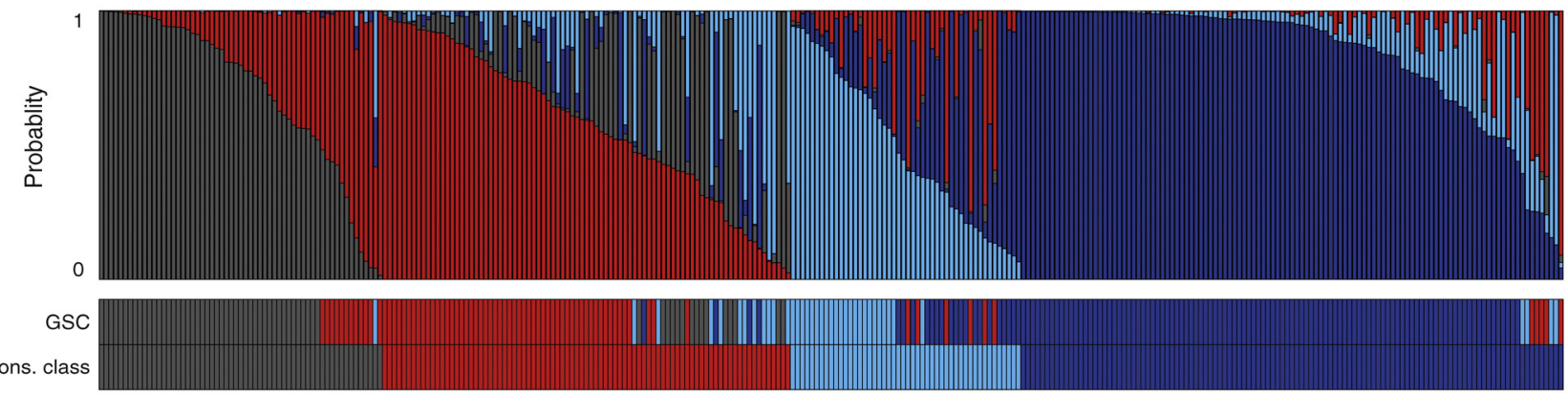

\section{C}
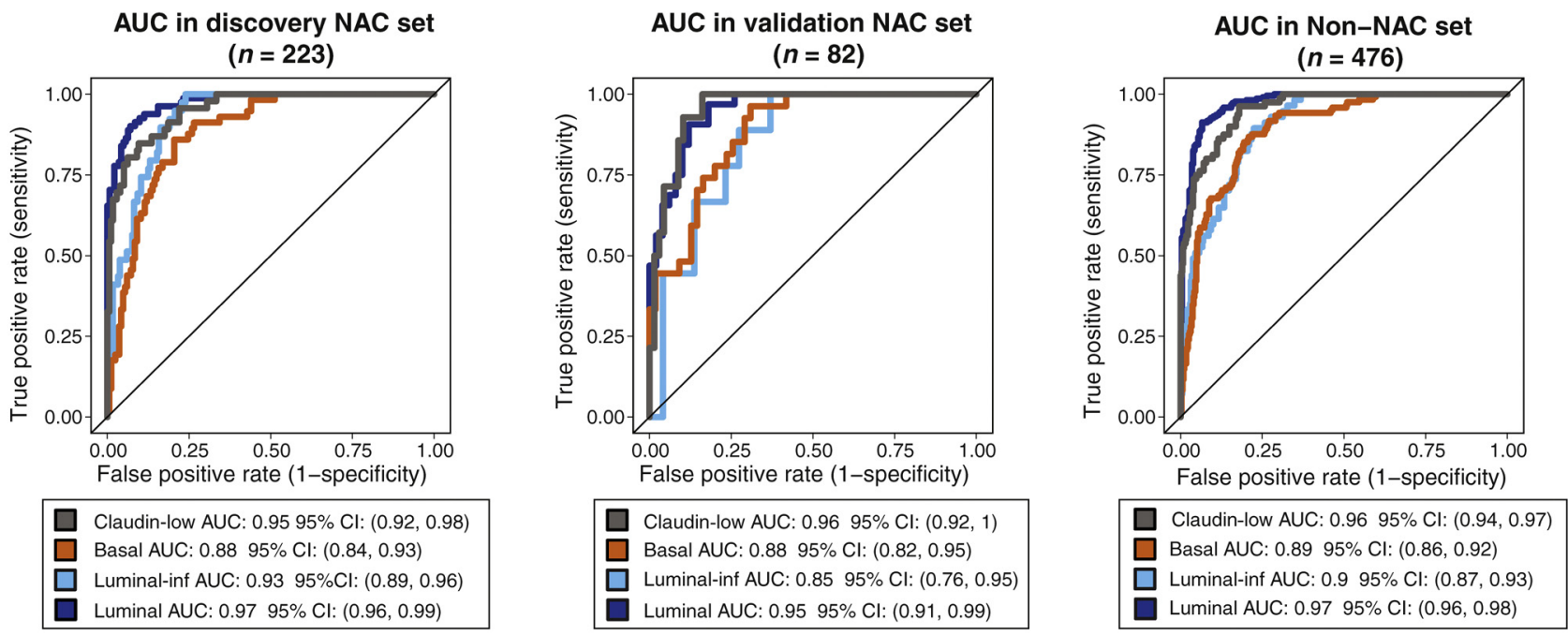

Fig. 3 - Discovery and validation of the GSC. (A) Proposed GSC bladder cancer classes derived from a consensus of four models (UNC, MDA, TCGA, and Lund). Colors indicate each class: claudin-low (gray), basal (red), luminal-infiltrated (light blue), and luminal (dark blue). (B) GSC cross-validation scores for each sample in the NAC dataset. The vertical bands represent the probability of each sample belonging to each class. The bottom bars indicate the classes predicted by GSC as well as the consensus classes. (C) Performance of the GSC in the discovery (10-fold cross validation for model performance) and two independent validation cohorts (NAC validation cohort and non-NAC validation cohort). Across all the cohorts, GSC was able to predict all subtypes significantly with a high area under the curve (compared with consensus classes). AUC = area under the curve; $\mathrm{CI}=$ confidence interval; Cons. = consensus; GSC = genomic subtyping classifier; inf = infiltrated; MDA = MD Anderson Cancer Center; NAC = neoadjuvant chemotherapy; TCGA = The Cancer Genome Atlas; UNC = University of North Carolina.

dataset that combined patients from TCGA, MDA, and the University of Lund datasets $(n=476)$. We validated the relative changes in outcome by GSC subtype in patients treated with and without NAC (Fig. 4A). Patients with GSC basal tumors had a $3-\mathrm{yr}$ OS rate of $49.2 \%$ (95\% confidence interval $[\mathrm{CI}] 39.5-61.2 \% ; p<0.001)$ in the non-NAC cohort compared with $77.8 \%(95 \% \mathrm{CI} 67.2-90.0 \% ; p<0.001)$ in the NAC cohort.

had the worst outcome, the prognosis of patients with basal tumors significantly improved when treated with NAC. (B) OS stratified according to the MDA subtypes. Patients with MDA luminal tumors had the best outcome in both the non-NAC (left) and NAC (right) settings. In the presence of NAC, patients with p53-like tumors had a significantly shorter $O S$ when compared with patients with MDA luminal tumors. (C) OS stratified according to the TCGA clusters. Clusters I and II clearly subdivide luminal tumors into two subsets-a subset with good prognosis (cluster I) and a subset with poor prognosis (cluster II)-although neither was affected by NAC. Basal tumors were subdivided into two subsets with similar prognosis in the non-NAC setting, but discrepant responses to NAC. The OS of patients with cluster III tumors was superior when treated with NAC, whereas that of patients with cluster IV tumors was poor regardless of NAC. (D) OS stratified according to the Lund subtypes. Patients with luminal tumors (Uro A and genomically unstable) had the best outcome without (left) and with (right) NAC. The OS of patients with basal tumors (Uro B and SCC-like) was inferior to that of Uro A tumors in the absence of NAC (left). However, with NAC (right) the outcome was similar to that in Uro A patients. The $p$ values represent Cox proportional hazard ratios. MDA = MD Anderson Cancer Center; NAC = neoadjuvant chemotherapy; $0 S$ = overall survival; $S C C=$ squamous cell carcinoma; TCGA = The Cancer Genome Atlas; UNC = University of North Carolina; Uro = urobasal. 
A

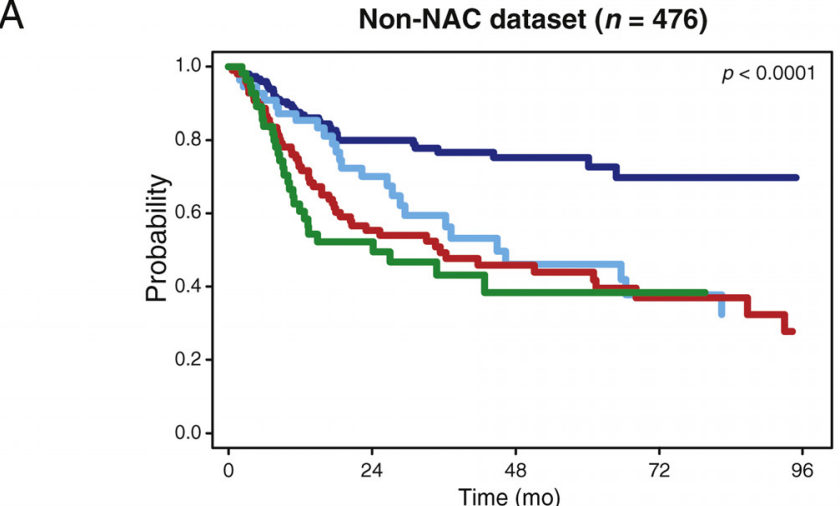

45

86
30
19
42

45
12

12
7

25
Numbers at risk

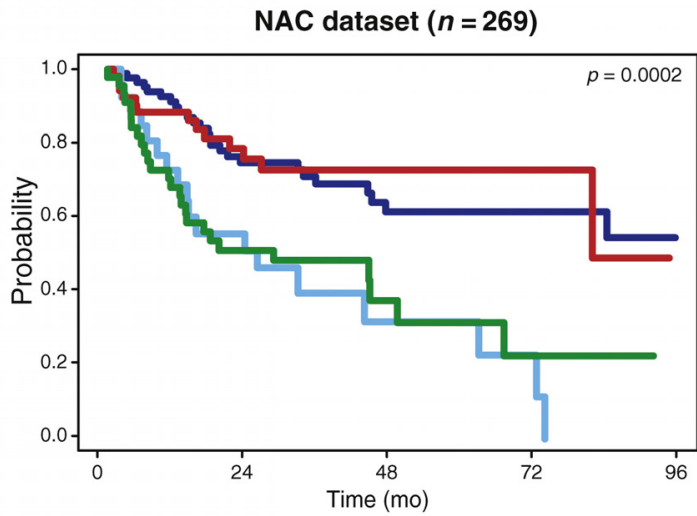

$\begin{array}{rrrrr}\square 55 & 28 & 10 & 5 & 1 \\ \square 67 & 35 & 17 & 6 & 2 \\ \square 33 & 16 & 7 & 5 & 2 \\ \square 107 & 59 & 33 & 16 & 8\end{array}$

Numbers at risk

B
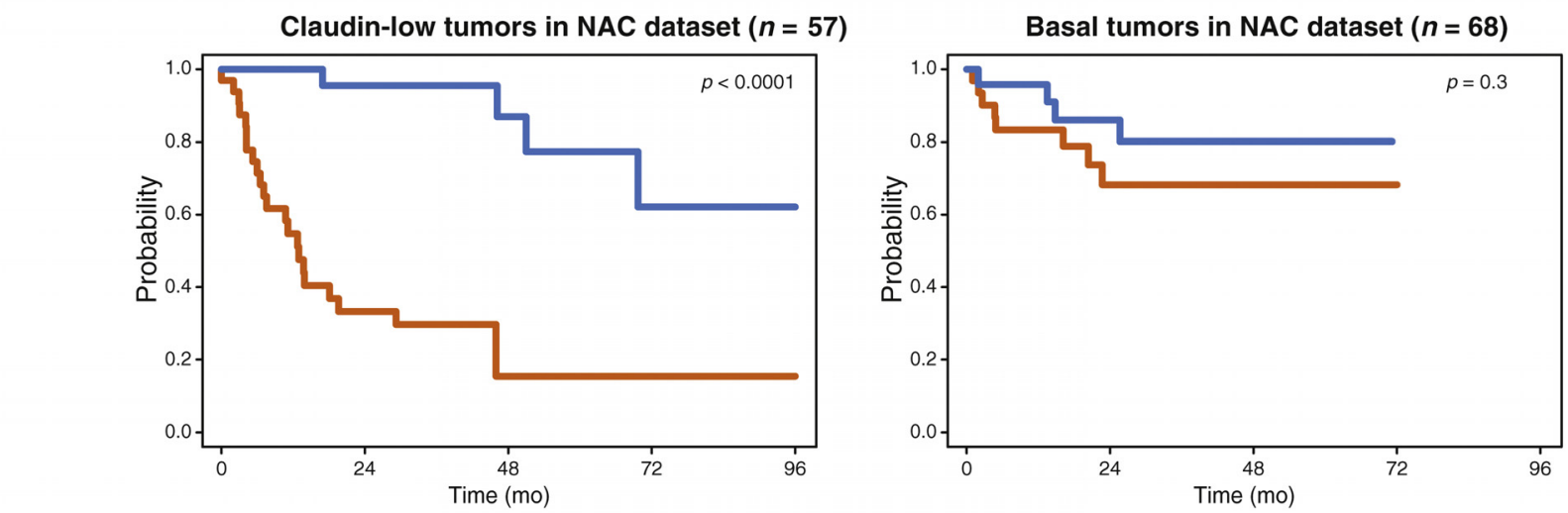

Response $\square 23$ Nonresponse $\square 32$

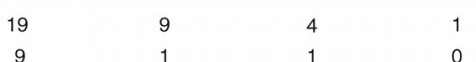

Numbers at risk

$\square 28$

$\square 37$

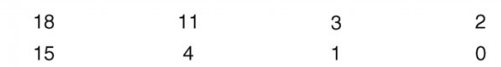

Numbers at risk

Luminal-inf tumors in NAC dataset $(n=33)$
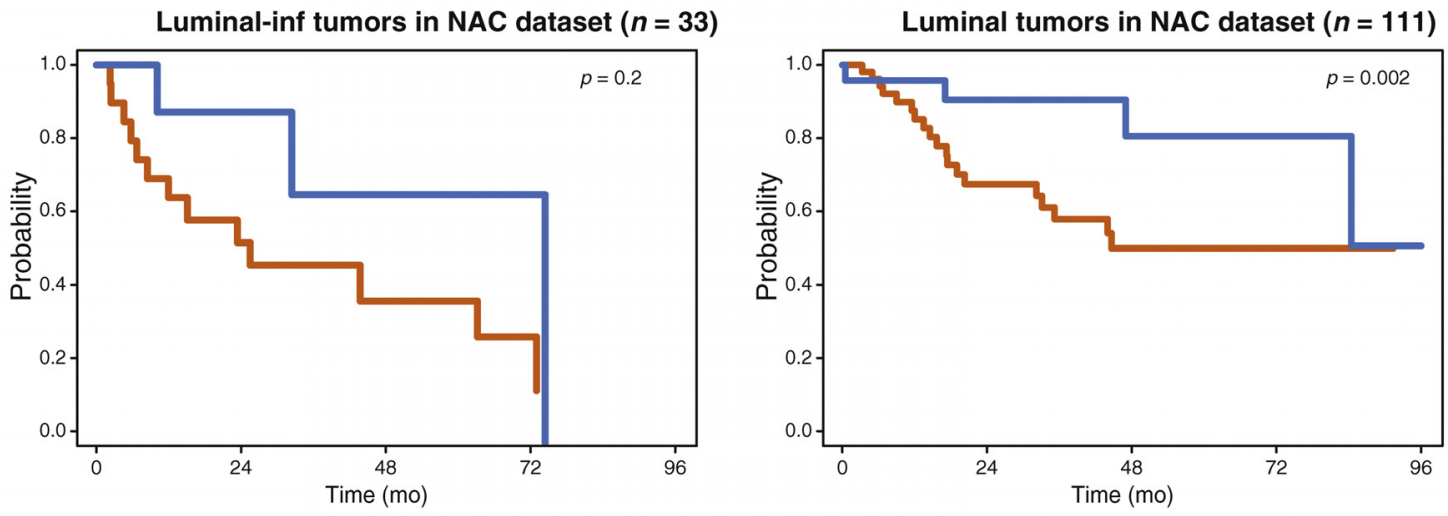

\footnotetext{
Response $\square 9$
}

Nonresponse $\square 20$

6

$$
2
$$

Numbers at risk $\square 48$
$\square 54$

31
25

31
25

Numbers at risk

Fig. 4 - Clinical significance of GSC with and without cisplatin-based NAC. (A) Kaplan-Meier plots for OS in non-NAC (left) and NAC (right) datasets stratified according to the classes predicted by GSC. (B) OS of the NAC dataset according to major pathological downstaging stratified by claudin-low (upper left), basal (upper right), luminal-infiltrated (lower left), and luminal (lower right) subtypes. GSC = genomic subtyping classifier; inf = infiltrated; NAC = neoadjuvant chemotherapy; $0 S=$ overall survival. 
Table 2 - Results of Cox proportional hazard analysis of GSC's ability to predict overall survival (NAC and non-NAC)

\begin{tabular}{|c|c|c|c|c|c|c|c|c|c|}
\hline \multirow[t]{3}{*}{ Variable } & \multicolumn{3}{|c|}{ MVA } & \multicolumn{3}{|c|}{ MVA $^{a}$} & \multicolumn{3}{|c|}{ MVA $^{a}$} \\
\hline & \multicolumn{3}{|c|}{ Non-NAC set $(n=476)$} & \multicolumn{3}{|c|}{ All NAC set $(n=269)$} & \multicolumn{3}{|c|}{ NAC validation set $(n=69)$} \\
\hline & HR & $95 \% \mathrm{CI}$ & $p$ value & HR & $95 \% \mathrm{CI}$ & $p$ value & HR & $95 \% \mathrm{CI}$ & $p$ value \\
\hline Age & 1.02 & $1-1.05$ & 0.066 & 1.01 & $0.99-1.04$ & 0.235 & 0.98 & $0.93-1.03$ & 0.433 \\
\hline Female (Ref) & 1 & - & 1.000 & 1 & - & 1.000 & 1 & - & 1.000 \\
\hline Male & 0.81 & $0.53-1.24$ & 0.330 & 1.12 & $0.67-1.86$ & 0.661 & 4.38 & $0.7-27.52$ & 0.115 \\
\hline Luminal (Ref) & 1 & - & 1.000 & 1 & - & 1.000 & 1 & - & 1.000 \\
\hline Inf-luminal & 2.38 & $1.33-4.28$ & 0.004 & 2.46 & $1.29-4.7$ & 0.006 & 5.68 & $0.4-81.3$ & 0.201 \\
\hline Basal & 2.22 & $1.34-3.68$ & 0.002 & 0.84 & $0.42-1.68$ & 0.614 & 0.88 & $0.16-4.94$ & 0.881 \\
\hline Claudin-low & 3.06 & $1.71-5.47$ & $<0.001$ & 2.16 & $1.22-3.81$ & 0.008 & 3.73 & $0.81-17.25$ & 0.092 \\
\hline
\end{tabular}

In multivariable analysis adjusting for clinical tumor stage, age, and gender in the non-NAC cohort, patients with a GSC basal subtype had a hazard ratio of $2.22(p=0.002$; Table 2) for OS compared with the luminal subtype. In contrast, in the NAC cohort, GSC basal subtype patients did not fare differently from patients with luminal tumors (hazard ratio: $0.84, p=0.61$; Table 2 ). Similar results were observed when considering only those patients from the independent NAC dataset $(n=69)$ not used for GSC model training (Table 2). Overall, this analysis suggested that the outcome of patients with basal tumors may improve the most with NAC.

3.6. NAC survival benefit in GSC basal tumors is independent of pathological response

As in our analysis using the previously described subtyping methods, GSC was not significantly associated with a major pathological response (ie, ypT $<2 \mathrm{NO}$ ) in the NAC cohort (Supplementary Tables 3 and 5), even though the major pathological response was associated with improved OS $(p<0.001$; Supplementary Fig. 7 and Supplementary Table 6). In an exploratory analysis, we further compared NAC responders $(n=108)$ and nonresponders $(n=143)$ in each GSC subtype (Fig. 4B). Patients with luminal tumors who experienced a major response $(n=48)$ had a $3-y r$ OS of 95\% (95\% CI 89-100\%) compared with 58\% (95\% CI 44-76\%) in nonresponders $(n=54, p=0.002$; Fig. $4 \mathrm{~B}$, lower right). In stark contrast, patients with GSC basal tumors did not show any significant differences in OS between major responders and nonresponders (Fig. 4B, upper right and Supplementary Table 7). The findings in the luminal-infiltrated and claudinlow tumors were more similar to the luminal tumors, although the sample sizes of these subgroups were too small and the duration of follow-up too short to allow definitive conclusions (Fig. 4B, left panels and Supplementary Table 8).

\section{Discussion}

The recent molecular characterization of MIBC, including the description of subtypes based on gene expression [10-13], provides a framework for further study of this frequently lethal malignancy and offers potential biological insight into different clinical phenotypes. One potential immediate impact of the molecular subtyping is to guide selection of optimal therapy. This concept was first suggested by Choi et al [11] in the context of cisplatin-based NAC, and subsequently by two trials in the context of systemic checkpoint inhibition $[18,19]$. Here, we have provided more evidence in a large patient cohort that outcome after NAC varies by molecular subtype. Furthermore, we have developed the methodology for single patient subtyping in a CLIAcertified laboratory [16], which represents a significant step toward potential clinical application of molecular subtyping.

The most important finding of our study was the relative shift in outcome by subtype in patients with and without NAC, albeit based on a comparison across studies. Our findings support the clinical utility of the four subtypes. Patients with basal tumors appear to derive the most benefit from NAC. These highly proliferative basal tumors demonstrated a poor prognosis when treated with surgery alone [11], but their prognosis was dramatically improved in the NAC cohort. Patients with luminal nonimmuneinfiltrated tumors had the best prognosis, irrespective of the treatment strategy, implying that these patients do not appear to derive benefit from NAC. The pathological stage and prognosis of patients with luminal immune-infiltrated tumors were significantly worse than those with luminal noninfiltrated tumors. Patients with luminal-infiltrated tumors appear to have poor prognosis with and without NAC.

Of a potentially high clinical impact, the patients with luminal-infiltrated tumors (corresponding to TCGA cluster II) seemed to benefit most from checkpoint inhibition with azetolizumab in the IMvigor 210 trial [18]. However, in the recently published CheckMate 275 trial, patients with basal tumors appeared to benefit from nivolumab, another checkpoint inhibitor [19]. Unfortunately, the gene expression data from neither of these trials have been made publicly available and the methodology for assignment of patients to TCGA clusters has not been revealed. Therefore, it is not currently possible to draw conclusions on the impact of subtyping on response to checkpoint blockade. Clinical trials will need to address the relative merit of NAC and perioperative checkpoint inhibition in these patients. 
We recapitulated similarities between the most recently discovered claudin-low tumors and TCGA cluster IV $[10,15]$. However, while Kardos et al [15] found claudinlow tumors to be evenly distributed between clusters III and IV, in our dataset claudin-low tumors were enriched in cluster IV (75\%). Both clusters III and IV are basal tumors with a strong mesenchymal signature, and both show the highest degree of immune infiltration. Patients with claudin-low tumors had the worst prognosis irrespective of treatment strategy in our analysis, suggesting also that these patients derived little or no benefit from NAC. Importantly, although these tumors showed the highest rate of immune infiltration, the benefit of checkpoint inhibition was limited in this subtype in the IMvigor 210 and the CheckMate 275 trial $[18,19]$. These patients should be targeted with priority for inclusion in clinical trials of novel agents.

Although we observed clear relationships between OS after NAC and molecular subtypes, we did not observe a clear effect on pathological response to NAC. The lack of this expected association [4] may simply be due to sample size and patient selection, as well as surgical downstaging with TUR alone. However, our findings resemble those in breast cancer, where pathological response relates to outcome in some, but not all, molecular subtypes [20]. In our series, the lack of association between pathological response and OS was observed primarily in the basal tumors. Prospective validation in a larger cohort is required to resolve this issue in MIBC.

Limitations of this study include the retrospective design. Moreover, our analysis is also confounded by comparisons between patient cohorts from different studies. This makes validation in prospectively collected cohorts necessary. Since the clinical characteristics of the cohorts used were different and an unknown proportion of the Lund dataset was not treated with a curative intent, we were not able to match non-NAC with NAC cohorts to perform a direct comparison of the impact of NAC between subtypes. Nonetheless, the relative differences in OS between subtypes in the NAC and non-NAC settings suggest a differential impact of the subtypes on outcome after NAC.

\section{Conclusions}

We provide the most compelling data to date that suggest a relationship between molecular subtypes and response to cisplatin-based NAC in MIBC. With or without NAC, patients with luminal tumors do well, implying that NAC is perhaps unnecessary in this subtype. Immune-infiltrated luminal tumors appear to have limited benefit from NAC, but have been shown previously to respond best to checkpoint inhibition, suggesting an alternative for systemic therapy in these patients. Claudin-low tumors have the worst outcome regardless of NAC treatment, and novel therapies are urgently needed for this patient cohort. The impact of NAC on OS was greatest in patients with basal tumors, which raises the hypothesis that these patients should be prioritized for NAC. These findings require prospective validation before this single-sample classifier can be used in clinical practice.

Author contributions: Peter C. Black had full access to all the data in the study and takes responsibility for the integrity of the data and the accuracy of the data analysis.

Study concept and design: Seiler, Wright, van der Heijden, Douglas, Dall'Era, Boormans, Davicioni, Black.

Acquisition of data: Seiler, Erho, Palmer-Aronsten, Buerki, Lam, Davicioni, Black.

Analysis and interpretation of data: Seiler, Erho, Ashab, Choeurng, Wang, Choi, McConkey, Kardos, Sjödahl, Hoadley, Kim, Black.

Drafting of the manuscript: Seiler, Erho, Wang, Choeurng, Davicioni, Ashab, Black.

Critical revision of the manuscript for important intellectual content: Winters, Douglas, van Rhijn, van de Putte, Kardos, Todenhöfer, Kiss, Buerki, Choi, McConkey, Sjödahl, Hoadley, Lerner, Van Kessel, Zwarthoff, North, Crabb, Sommerlad, Thalmann, Davicioni, Kim, Wright, van der Heijden, Dall'Era.

Statistical analysis: Wang, Choeurng.

Obtaining funding: Davicioni.

Administrative, technical, or material support: Buerki, Lam, PalmerAronsten, Davicioni.

Supervision: Black.

Other: None.

Financial disclosures: Peter C. Black certifies that all conflicts of interest, including specific financial interests and relationships and affiliations relevant to the subject matter or materials discussed in the manuscript (eg, employment/affiliation, grants or funding, consultancies, honoraria, stock ownership or options, expert testimony, royalties, or patents filed, received, or pending), are the following: Hussam Al Deen Ashab: Employment GenomeDx. Nicholas Erho: Employment, GenomeDx. Natalie Q. Wang: Employment, GenomeDx. Voleak Choeurng: Employment, GenomeDx. Ewan A. Gibb: employment, GenomeDx. Beatrix Palmer-Aronsten: employment, GenomeDx. Lucia L. Lam: employment, GenomeDx. Christine Buerki: employment, GenomeDx. Elai Davicioni: employment, GenomeDx. Seth P Lerner: consulting or advisory role: Oncogenex, Sitka, Nucleix, Vaxiion, Taris BioMedical, Telesta Therapeutics, UroGen, Ferring. David J. McConkey: stock or other ownership: ApoCell; patents, royalties, other intellectual property: Pending patent_ UTSC.P1206US. Woonyoung Choi: patents, royalties, other intellectual property: pending: Methods of characterizing and treating molecular subset of muscle-invasive bladder cancer. William Y. Kim: patents, royalties, other intellectual property: pending: BASE47. Simon J. Crabb: honoraria: Bayer, Astellas Pharma, Janssen; consulting or advisory role: Bayer, Sanofi, Astellas Pharma, Janssen, Pfizer, Roche. Scott North: honoraria: Janssen-Ortho, Astellas Pharma, Novartis, Pfizer, Sanofi Canada; consulting or advisory role: Janssen Oncology, Astellas Pharma, Novartis, Sanofi Canada, Pfizer, Roche Canada, Merck, AstraZeneca. Ellen C. Zwarthoff: honoraria: MDxHealth; patents, royalties, other intellectual property: Erasmus MC. Michiel S. van der Heijden: consulting or advisory role: Roche/Genentech, Astellas Pharma, AstraZeneca/MedImmune; travel, accommodations, expenses: Novartis, Astellas Pharma, MSD Oncology. Peter C. Black: consulting or advisory role: Abbvie, Astellas Pharma, Janssen Oncology, Amgen, Novartis, BioCancell, Sitka, Cubist, Bayer, Merck, Sanofi Canada, Biosyent, Ferring, Lilly, Roche Canada, Spectrum Pharmaceuticals; patents, royalties, other intellectual property: 1. PCT/CA2014/000787. Canada. 2014-11-03 Cancer Biomarkers and Classifiers and uses thereof; 2. \#61899648 United States. 201303-13 Bladder cancer signature. 
Funding/Support and role of the sponsor: Roland Seiler: salary funded by the Swiss National Foundation. GenomeDx funded gene expression analysis. Peter C. Black: AUA Foundation/Astellas Rising Stars in urology award. Bas W.G. van Rhijn: The EAU-European Urological Scholarship Programme. Michiel S. van der Heijden: Veni grant, Netherlands Organisation for Scientific Research. The sponsors played a role in the design and conduct of the study, and in collection, management, analysis, and interpretation of the data.

Acknowledgments: We thank the Core Facility for Molecular Pathology \& Bio-banking of the Netherlands Cancer Institute;1;-Antoni van Leeuwenhoek for their assistance.

\section{Appendix A. Supplementary data}

Supplementary data associated with this article can be found, in the online version, at http://dx.doi.org/10.1016/j. eururo.2017.03.030.

\section{References}

[1] Bajorin DF, Herr HW. Kuhn's paradigms: are those closest to treating bladder cancer the last to appreciate the paradigm shift? J Clin Oncol 2011;29:2135-7.

[2] Grossman HB, Natale RB, Tangen CM, et al. Neoadjuvant chemotherapy plus cystectomy compared with cystectomy alone for locally advanced bladder cancer. N Engl J Med 2003;349:859-66.

[3] International Collaboration of Trialists, Medical Research Council Advanced Bladder Cancer Working Party, European Organisation for Research and Treatment of Cancer Genito-Urinary Tract Cancer Group, et al. International phase III trial assessing neoadjuvant cisplatin, methotrexate, and vinblastine chemotherapy for muscle-invasive bladder cancer: long-term results of the BA06 30894 trial. J Clin Oncol 2011;29:2171-7.

[4] Zargar H, Espiritu PN, Fairey AS, et al. Multicenter assessment of neoadjuvant chemotherapy for muscle-invasive bladder cancer. Eur Urol 2015;67:241-9.

[5] Groenendijk FH, de Jong J, Fransen van de Putte EE, et al. ERBB2 mutations characterize a subgroup of muscle-invasive bladder cancers with excellent response to neoadjuvant chemotherapy. Eur Urol 2016;69:384-8.

[6] Liu D, Plimack ER, Hoffman-Censits J, et al. Clinical validation of chemotherapy response biomarker ERCC2 in muscle-invasive urothelial bladder carcinoma. JAMA Oncol 2016;2:1094-6.
[7] Plimack ER, Dunbrack RL, Brennan TA, et al. Defects in DNA repair genes predict response to neoadjuvant cisplatin-based chemotherapy in muscle-invasive bladder cancer. Eur Urol 2015;68:959-67.

[8] Van Allen EM, Mouw KW, Kim P, et al. Somatic ERCC2 mutations correlate with cisplatin sensitivity in muscle-invasive urothelial carcinoma. Cancer Discov 2014;4:1140-53.

[9] Biton A, Bernard-Pierrot I, Lou Y, et al. Independent component analysis uncovers the landscape of the bladder tumor transcriptome and reveals insights into luminal and basal subtypes. Cell Rep 2014;9:1235-45.

[10] Cancer Genome Atlas Research Network. Comprehensive molecular characterization of urothelial bladder carcinoma. Nature 2014;507: 315-22.

[11] Choi W, Porten S, Kim S, et al. Identification of distinct basal and luminal subtypes of muscle-invasive bladder cancer with different sensitivities to frontline chemotherapy. Cancer Cell 2014;25: 152-65.

[12] Damrauer JS, Hoadley KA, Chism DD, et al. Intrinsic subtypes of high-grade bladder cancer reflect the hallmarks of breast cancer biology. Proc Natl Acad Sci U S A 2014;111:3110-5.

[13] Sjodahl G, Lauss M, Lovgren K, et al. A molecular taxonomy for urothelial carcinoma. Clin Cancer Res 2012;18:3377-86.

[14] Volkmer JP, Sahoo D, Chin RK, et al. Three differentiation states riskstratify bladder cancer into distinct subtypes. Proc Natl Acad Sci U S A 2012;109:2078-83.

[15] Kardos J, Chai S, Mose LE, et al. Claudin-low bladder tumors are immune infiltrated and actively immune suppressed. JCI Insight 2016;1:e85902

[16] Erho N, Crisan A, Vergara IA, et al. Discovery and validation of a prostate cancer genomic classifier that predicts early metastasis following radical prostatectomy. PLoS One 2013;8:e66855.

[17] Piccolo SR, Sun Y, Campbell JD, Lenburg ME, Bild AH, Johnson WE. A single-sample microarray normalization method to facilitate personalized-medicine workflows. Genomics 2012;100:337-44.

[18] Rosenberg JE, Hoffman-Censits J, Powles T, et al. Atezolizumab in patients with locally advanced and metastatic urothelial carcinoma who have progressed following treatment with platinum-based chemotherapy: a single-arm, multicentre, phase 2 trial. Lancet 2016;387:1909-20.

[19] Sharma P, Retz M, Siefker-Radtke A, et al. Nivolumab in metastatic urothelial carcinoma after platinum therapy (CheckMate 275): a multicentre, single-arm, phase 2 trial. Lancet Oncol 2017;18:312-22.

[20] von Minckwitz G, Untch M, Blohmer JU, et al. Definition and impact of pathologic complete response on prognosis after neoadjuvant chemotherapy in various intrinsic breast cancer subtypes. J Clin Oncol 2012;30:1796-804. 